\title{
Bulk versus surface contributions to the Rashba spin splitting of Shockley surface states
}

\author{
H. Ishida \\ College of Humanities and Sciences, Nihon University, Tokyo 156-8550, Japan
}

(Received 29 July 2018; published 19 November 2018)

\begin{abstract}
Shockley surface states of a bulk crystal with both time reversal and space inversion symmetries exhibit the Rashba spin splitting due to the broken space inversion symmetry at the surface. Since the evanescent states in the bulk region are doubly degenerate with respect to spin degrees of freedom even in the presence of spin-orbit interaction (SOI), one might think that the entire spin splitting occurs via the SOI in the surface region where the potential energy deviates from the bulk one. In the present work, we elucidate why this is not the case. Namely, in the presence of SOI, the complex energy bands are modified such that a pair of evanescent states having the same energy and the same complex wave number become two distinct solutions of the Schrödinger equation that do not satisfy the same boundary condition. Since the tail of the surface-state wave function is expressed as a superposition of these evanescent waves, the bulk region also contributes to the Rashba spin splitting. We illustrate this effect by a first-principles calculation for $\mathrm{Au}(111)$ and by a simplified $s p$-band model Hamiltonian on a square lattice.
\end{abstract}

DOI: 10.1103/PhysRevB.98.205412

\section{INTRODUCTION}

The spin degeneracy of electronic states is lifted at the surface of nonmagnetic crystals having space inversion symmetry. This effect is called Rashba spin splitting, since the energy dispersion of the $L$-gap surface state on $\mathrm{Au}(111)$, for which this effect was first observed [1], was well fitted by the Bychkov-Rashba Hamiltonian for two-dimensional (2D) electrons exposed to a vertical electric field [2]. However, Petersen and Hedegård [3] pointed out that the spin splitting arising from the spin-orbit interaction (SOI) involving the normal derivative of surface potential barrier is by several orders of magnitude smaller than the experimental one in the case of $\mathrm{Au}(111)$. Furthermore, by using a tight-binding Hamiltonian comprising three $p$ orbitals, they demonstrated that the spin splitting of surface bands arises from the conventional SOI of the type $\hat{H}_{s o}=2 \lambda(\vec{l} \cdot \vec{s})(\vec{l}$ and $\vec{s}$ are orbital and spin angular momentum operators of constituent atoms, respectively), if the hopping between the neighboring $p_{z}$ (the $z$ axis is the surface normal) and $p_{x, y}$ orbitals is allowed due to the potential asymmetry in the $z$ direction. This was consistent with the density-functional-theory (DFT) calculation of Nicolay et al. [4] that reproduced the spin-split surface bands on $\mathrm{Au}(111)$ quite well only with the aforementioned conventional SOI term. Subsequently, a number of mechanisms were proposed in order to account for large energy splittings observed for clean surfaces [5-8] as well as for those covered with heavy atoms such as $\mathrm{Pb}$ and $\mathrm{Bi}$ [9-12]. Bihlmayer et al. [13] and Nagao et al. [14] demonstrated that it is not the asymmetry

Published by the American Physical Society under the terms of the Creative Commons Attribution 4.0 International license. Further distribution of this work must maintain attribution to the author(s) and the published article's title, journal citation, and DOI. of the potential gradient but the asymmetry of the surfacestate wave function (or charge density) that determines the size of the spin splitting. Park et al. [15] and Kim et al. [16] suggested that the orbital angular momentum induced in surface-state wave functions plays a central role in the formation of helically polarized spin textures in spin-split surface bands. More recently, Krasovskii proposed that the Rashba spin splitting is caused by the relativistic modification of surface-state wave functions [17]. Yan et al. suggested that the $L$-gap surface states on noble metals are of topological nature [18].

In the present work, we present a novel viewpoint on the physical mechanism of the Rashba spin splitting. It differs from the aforementioned ones in that it sheds light on the role of the bulk electronic states. To explain the motivation for the present work clearly, we show in Fig. 1(a) the spin splitting of the $L$-gap surface state on $\mathrm{Au}(111)$ as a function of the wave number along the $\bar{\Gamma}-\bar{M}$ line (chosen as the $x$ direction) calculated by a DFT code [19] that combines the full-potential linearized augmented plane wave (LAPW) method [20] and the embedding technique of Inglesfield [21,22]. In the embedding method, the space is partitioned into the surface region with a finite thickness and the semi-infinite bulk, and the Green's function in the surface region, is calculated by representing the effects of the semi-infinite substrate via a complex embedding potential acting on the boundary surface between the two regions. In Fig. 1(a), $L$, the number of the Au layers in the embedded surface region, is varied from $L=1$ to 7 . It is seen that the convergence of the energy splitting of the two surface bands, $\Delta \varepsilon$, with $L$ is surprisingly fast, and even the calculation with $L=1$, in which the second and deeper Au layers are represented by the embedding potential, can reproduce $\Delta \varepsilon$ very well in a wide $k_{x}$ range. On the one hand, this implies that the screening length of potential energies is short in a metal with high electron densities. On the other hand, however, this does not indicate that the surface-state wave function is 

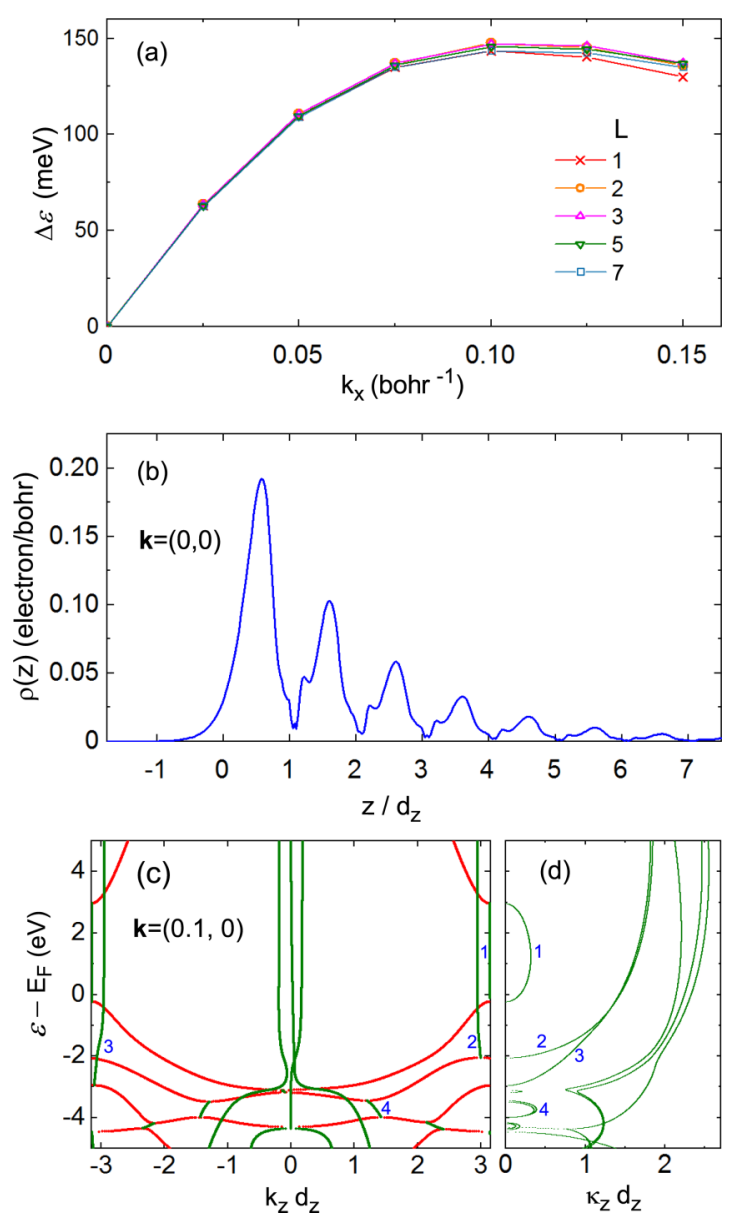

FIG. 1. (a) Spin splitting $\Delta \varepsilon$ of the $L$-gap surface state on $\mathrm{Au}(111)$ as a function of $k_{x}$, wave number along the $\bar{\Gamma}-\bar{M}$ line. $L$ denotes the number of $\mathrm{Au}$ layers included in the embedded surface region. (b) Planar-averaged charge density of the $L$-gap surface state on $\mathrm{Au}(111)$ at $\bar{\Gamma}$ as a function of normal coordinate $z . d_{z}$ denotes the layer spacing between two neighboring $\mathrm{Au}(111)$ layers, and $z / d_{z}=i$ $(i=1,2, \ldots, 7)$ indicates the positions of $\mathrm{Au}$ atomic planes. (c) Complex band structure of $\mathrm{Au}(111)$ at $k_{x}=0.1 \mathrm{bohr}^{-1}$ along the $\bar{\Gamma}-\bar{M}$ line. Left and right panels show, respectively, the real and imaginary part of the $z$ component of wave number $q=k_{z}+i \kappa_{z}$. Red lines indicate Bloch states with $\kappa_{z}=0$, while pairs of green lines numbered as $1,2,3, \ldots$ in both panels represent evanescent states with $\kappa_{z}>0$. For brevity, only a few bands are numbered. The decay length of the $L$-gap surface state is determined by the $\kappa_{z}$ value of the band "1." Energy is measured relative to the Fermi energy $E_{F}$.

strongly localized within the first Au layer. Figure 1(b) shows the planar-averaged charge density of the $L$-gap state at $\bar{\Gamma}$ as a function of the normal coordinate $z$. Surely the charge density decays exponentially toward the interior of the metal. However, it has considerable amplitudes on the second and deeper layers. Indeed, according to Bihlmayer et al. [13], more than 40 percent of $\Delta \varepsilon$ arises from the SOI in the second and deeper Au layers. In the present calculation with $L=1$, this contribution is represented by the embedding potential. For a given energy $\varepsilon$ and planar wave vector $\mathbf{k}$, the embedding potential is calculated from the wave functions of generalized Bloch states (complex energy bands) with complex wave number in the normal direction, $q=k_{z}+i \kappa_{z}$, which decay or propagate toward the interior of the metal. The energy versus $q$ plot of the complex energy bands is called the complex band structure [23-25]. In Fig. 1(c) we show the complex band structure of $\mathrm{Au}(111)$ at $\mathbf{k}=(0.1,0) \mathrm{bohr}^{-1}$ around which $\Delta \varepsilon$ becomes the largest. As is seen, not only the Bloch states with $\kappa_{z}=0$ (red lines) but also all evanescent states with $\kappa_{z}>0$ (green lines) are doubly degenerate with respect to spin degrees of freedom. Then, as a natural consequence, one may raise a question as to why the embedding potential calculated from the doubly degenerate complex energy bands is able to represent the contribution of the bulk region to the Rashba spin splitting of the surface bands. This is the central question clarified in the present work.

The complex band structure in a bulk crystal with both time reversal and space inversion symmetries is doubly degenerate. When electronic states are doubly degenerate, one may tend to assume that the two states are related by some symmetry operator. Indeed, regarding Bloch states, by applying both time reversal and space inversion operators to one of the two states in a degenerate pair, one obtains the other solution in the pair. In clear contrast, regarding evanescent waves, as will be shown later, there exist no simplified mathematical operators relating the two states in a degenerate pair when SOI is present. Instead, they become two distinct solutions of the Schrödinger equation that do not satisfy the same boundary condition at the boundary surface between the surface and bulk regions. Since the embedding potential is constructed from the wave functions of the evanescent states within a surface-projected bulk band gap, it can reproduce the bulk contributions to the spin splitting of surface bands.

The plan of the present paper is as follows. In Sec. II we formulate the complex energy bands (generalized Bloch states) of bulk crystals by using an orthonormal local-orbital basis set and discuss their properties when the system possesses both time reversal and space inversion symmetries. We also present a short review of the embedding theory and explain how the embedding potential of a semi-infinite substrate is constructed from the wave functions of generalized Bloch states. In Sec. III we illustrate the aforementioned Rashba spin splitting originating from the complex energy bands by a model calculation that employs a simplified $s p$ band Hamiltonian on a two-dimensional (2D) square lattice. In doing so, we explore two parameter regimes that can be distinguished from each other by the strength of SOI. Finally, we conclude in Sec. IV.

\section{THEORY}

In computing $\Delta \varepsilon$ in Fig. 1, we used the LAPW basis functions and the embedding theory of Inglesfield formulated in real space $[21,22]$. While the properties of the complex energy bands discussed in this section can be derived by using the real-space representation of electron wave functions, we switch to a formulation based on local-orbital basis functions in the following, since it greatly facilitates deriving the properties of complex energy bands.

\section{A. Complex band structure}

We consider a bulk crystal made by stacking atomic layers in the surface normal direction, which is chosen as the $z$ 
axis. We assume that two of the three primitive translation vectors of the crystal lie within the plane, while the third one connects equivalent atoms in two nearest-neighbor layers. To expand electron wave functions, we use a linear combination of atomic orbital (LCAO) type basis set $\left\{\left|\chi_{\ln }^{\mathbf{k}}\right\rangle\right\}$ consisting of basis functions localized in each layer, which are specified by the planar wave vector $\mathbf{k}$, integer layer index $l$, and index $n$ $(n=1,2, \ldots, N)$ that specifies atomic sites in the unit cell, types of atomic orbitals, and also electron spin [26]. The basis set is assumed to be orthonormal, i.e., $\left\langle\chi_{l n}^{\mathbf{k}} \mid \chi_{l^{\prime} n^{\prime}}^{\mathbf{k}}\right\rangle=\delta_{l, l^{\prime}} \delta_{n, n^{\prime}}$. Then $|\phi\rangle$, an arbitrary solution of the Schrödinger equation with $\mathbf{k}$ and real energy $\varepsilon$, is expanded in the form

$$
|\phi\rangle=\sum_{l, n}\left|\chi_{l n}^{\mathbf{k}}\right\rangle\left\langle\chi_{l n}^{\mathbf{k}} \mid \phi\right\rangle,
$$

where $\left\langle\chi_{\ln }^{\mathbf{k}} \mid \phi\right\rangle$ satisfies

$$
\hat{H}_{l, l+1}^{\mathbf{k}} \Phi(l+1)+\hat{H}_{l, l}^{\mathbf{k}} \Phi(l)+\hat{H}_{l, l-1}^{\mathbf{k}} \Phi(l-1)=\varepsilon \Phi(l) .
$$

Here, $\hat{H}_{l, l^{\prime}}^{\mathbf{k}}$ is the $N \times N$ transfer matrix whose $\left(n, n^{\prime}\right)$ element is given by $\left\langle\chi_{l n}^{\mathbf{k}}|\hat{H}| \chi_{l^{\prime} n^{\prime}}^{\mathbf{k}}\right\rangle$ with $\hat{H}$ being the one-electron Hamiltonian, $\hat{H}_{l, l^{\prime}}^{\mathbf{k}}$ is assumed to be nonvanishing only up to nearest-neighboring layers, and $\Phi(l)$ denotes a column vector of length $N$,

$$
\Phi(l)=\left(\left\langle\chi_{l 1}^{\mathbf{k}} \mid \phi\right\rangle,\left\langle\chi_{l 2}^{\mathbf{k}} \mid \phi\right\rangle, \ldots,\left\langle\chi_{l N}^{\mathbf{k}} \mid \phi\right\rangle\right)^{t} .
$$

Let us consider a generalized Bloch state with dimensionless complex wave number in the $z$ direction, $q=k_{z}+i \kappa_{z}$, for which $\Phi(l)$ can be expressed as

$$
\Phi(l)=e^{i q l} \mathbf{v}
$$

with a column eigenvector v. Inserting Eq. (4) into Eq. (2) yields

$$
[\hat{\mathcal{H}}(\mathbf{k}, q)-\varepsilon \hat{I}] \mathbf{v}=0,
$$

with the complex Hamiltonian,

$$
\hat{\mathcal{H}}(\mathbf{k}, q)=\hat{H}_{01}^{\mathbf{k}} e^{+i q}+\hat{H}_{00}^{\mathbf{k}}+\hat{H}_{10}^{\mathbf{k}} e^{-i q} .
$$

The complex band structure of the bulk crystal is obtained by plotting all complex wave numbers $q$ 's that satisfy Eq. (5), namely,

$$
\operatorname{det}[\hat{\mathcal{H}}(\mathbf{k}, q)-\varepsilon \hat{I}]=0,
$$

as a function of $\mathbf{k}$ and $\varepsilon$. At a given $(\mathbf{k}, \varepsilon)$, there are $2 N q$ 's that satisfy Eq. (7) and they can be computed, for example, by solving the eigenvalue problem of the $2 N \times 2 N$ transfer matrix [26-28].

For any $(\varepsilon, \mathbf{k}, q)$ satisfying Eq. (7), there exists also a left eigenvector $\mathbf{w}$ that fulfills

$$
\mathbf{w}^{\dagger}[\hat{\mathcal{H}}(\mathbf{k}, q)-\varepsilon \hat{I}]=0,
$$

whose hermitian conjugate gives

$$
\left[\hat{\mathcal{H}}\left(\mathbf{k}, q^{*}\right)-\varepsilon \hat{I}\right] \mathbf{w}=0 .
$$

For a Bloch state with $\kappa_{z}=0, \mathbf{w}=\mathbf{v}$ since $q=q^{*}$. On the other hand, for a positive $\kappa_{z}, e^{i q l} \mathbf{v}$ represents a solution decaying toward $z=+\infty$, while $e^{i q^{*} l} \mathbf{w}$ represents a solution decaying toward $z=-\infty$. In other words, two evanescent waves that share the same decaying constant and decay toward opposite directions appear as a pair regardless of the crystal symmetry. Hence, when $\varepsilon$ lies within a surface-projected bulk band gap at a given $\mathbf{k}$, the $2 N$ evanescent solutions are divided into those decaying toward $z=+\infty$ with complex wave numbers $\left\{q_{1}, q_{2}, \ldots, q_{N}\right\}$ and the corresponding eigenvectors $\left\{\mathbf{v}_{1}, \mathbf{v}_{2}, \ldots, \mathbf{v}_{N}\right\}$ and those decaying toward $z=-\infty$ with complex wave numbers $\left\{q_{1}^{*}, q_{2}^{*}, \ldots, q_{N}^{*}\right\}$ and the corresponding eigenvectors $\left\{\mathbf{w}_{1}, \mathbf{w}_{2}, \ldots, \mathbf{w}_{N}\right\}$.

So far, we assumed that the electron transfer is allowed only between nearest-neighboring layers. If $\hat{H}_{l, l^{\mathbf{k}}}^{\mathbf{k}}$ is nonvanishing for $\left|l-l^{\prime}\right| \leqslant l_{0}$, one may define the complex Hamiltonian as

$$
\hat{\mathcal{H}}(\mathbf{k}, q)=\sum_{l=-l_{0}}^{+l_{0}} \hat{H}_{0, l}^{\mathbf{k}} e^{+i(l q)},
$$

instead of Eq. (6). Then, the subsequent discussion holds true irrespective of the value of $l_{0}$, except that the number of the pairs of evanescent waves within a surface-projected bulk band gap becomes $l_{0} N$ instead of $N$. An alternative approach employed in previous works [26,27] is to redefine the unit cell by regarding a super cell comprising $l_{0}$ successive layers as a "single layer." Then, the interaction in the resultant Hamiltonian is limited to nearest-neighboring layers. In the present work, we adopt the latter and assume that $l_{0}=1$ in the following sections.

\section{B. Time reversal and space inversion}

Now, we consider a bulk crystal which is invariant with respect to both time reversal operator $\hat{\mathcal{T}}$ and space inversion operator $\hat{\mathcal{P}}$. By applying $\hat{\mathcal{P}}$ on a solution of the Schrödinger equation with real energy $\varepsilon$, complex wave vector $(\mathbf{k}, q)$, and eigenvector $\mathbf{v}$, we obtain another solution with energy $\varepsilon$, wave vector $(-\mathbf{k},-q)$, and eigenvector $\hat{\mathcal{P}} \mathbf{v}$. By further applying $\hat{\mathcal{T}}$ on this solution, we obtain a solution with energy $\varepsilon$, wave vector $\left(\mathbf{k}, q^{*}\right)$, and eigenvector $\hat{\mathcal{T}} \hat{\mathcal{P}} \mathbf{v}$. For Bloch states with vanishing $\kappa_{z}, \hat{\mathcal{T}} \hat{\mathcal{P}} \mathbf{v}$ represents a Bloch state which is orthogonal to $\mathbf{v}$ and has the same energy and the same wave vector as $\mathbf{v}$ because $q=q^{*}$. In other words, in a bulk crystal with both symmetries, Bloch states are always doubly degenerate and the two solutions are related to each other by $\hat{\mathcal{T}} \hat{\mathcal{P}}$.

This argument does not hold straightforwardly for an evanescent wave with complex wave vector $(\mathbf{k}, q)$ and eigenvector $\mathbf{v}$, since $\hat{\mathcal{T}} \hat{\mathcal{P}} \mathbf{v}$ having complex wave vector $\left(\mathbf{k}, q^{*}\right)$ represents an evanescent wave decaying toward the opposite direction. Nevertheless, as discussed in the preceding section, evanescent waves appear always as a pair, and for any evanescent wave with energy $\varepsilon$, wave vector $(\mathbf{k}, q)$, and eigenvector $\mathbf{v}$, there is a solution with energy $\varepsilon$, wave vector $\left(\mathbf{k}, q^{*}\right)$, and eigenvector $\mathbf{w}$. By applying $\hat{\mathcal{T}} \hat{\mathcal{P}}$ on the latter state, we obtain a solution with energy $\varepsilon$, wave vector $(\mathbf{k}, q)$, and eigenvector $\hat{\mathcal{T}} \hat{\mathcal{P}} \mathbf{w}$. Therefore, the complex energy bands of a bulk crystal with both time reversal and space inversion symmetries are doubly degenerate not only regarding Bloch states but also regarding evanescent states. However, in clear contrast to the case of Bloch states, the eigenvectors of two evanescent waves constituting a degenerate pair are $\mathbf{v}$ and $\hat{\mathcal{T}} \hat{\mathcal{P}}_{\mathbf{w}}$. Importantly, $\mathbf{v}$ and $\mathbf{w}$ are the right and left eigenvectors of the same complex Hamiltonian, and thus, in general, the two vectors are not 
related to each other by any simplified operator in the presence of SOI.

\section{Surface electronic structure}

Let us consider a semi-infinite crystal occupying the righthalf space. In its interior, the Hamiltonian matrix between nearest-neighboring layers and that between the same layers are given by $\hat{H}_{01}^{b, \mathbf{k}}\left[=\left(\hat{H}_{10}^{b, \mathbf{k}}\right)^{*}\right]$ and $\hat{H}_{00}^{b, \mathbf{k}}$, respectively, whereas the Hamiltonian matrices may deviate from the bulk ones near the surface. We divide the crystal into the surface layers with layer index $1 \leqslant l \leqslant L$ and the semi-infinite substrate with $l>L$. According to the local-orbital based embedding theory [22], the one-electron Green's function in the surface region $\left(1 \leqslant l, l^{\prime} \leqslant L\right)$ satisfies the equation,

$$
\sum_{l^{\prime \prime}=1}^{L}\left[\xi \hat{I} \delta_{l, l^{\prime \prime}}-\hat{H}_{l, l^{\prime \prime}}^{\mathbf{k}}-\hat{\Sigma}_{l, l^{\prime \prime}}^{\mathbf{k}}(\xi)\right] \hat{G}_{l^{\prime \prime}, l^{\prime}}^{\mathbf{k}}(\xi)=\hat{I} \delta_{l, l^{\prime}},
$$

where $\xi=\varepsilon+i \gamma$ is a complex energy, $\hat{I}$ is the identity matrix of order $N$, and $\hat{\Sigma}_{l, l^{\prime}}^{\mathbf{k}}$ and $\hat{G}_{l, l^{\prime}}^{\mathbf{k}}$ are $N \times N$ matrices whose $\left(n, n^{\prime}\right)$ element is given by $\left\langle\chi_{l n}^{\mathbf{k}}|\hat{\Sigma}| \chi_{l^{\prime} n^{\prime}}^{\mathbf{k}}\right\rangle$ and $\left\langle\chi_{l n}^{\mathbf{k}}|\hat{G}| \chi_{l^{\prime} n^{\prime}}^{\mathbf{k}}\right\rangle$, where $\hat{\Sigma}$ and $\hat{G}$ denote the local-orbital-based embedding potential (called more often lead self-energy [29,30]) and the Green's function, respectively. $\hat{\Sigma}_{l, l^{\prime}}^{\mathbf{k}}$ represents the effects of the semi-infinite substrate on the surface region. Since we assume that the transfer integral is nonvanishing only up to nearest-neighboring layers, $\hat{\Sigma}_{l, l^{\prime}}^{\mathbf{k}}$ vanishes except for the boundary element $[29,30]$,

$$
\hat{\Sigma}_{L, L}^{\mathbf{k}}(\xi)=\hat{H}_{L, L+1}^{\mathbf{k}} \hat{G}_{L+1, L+1}^{\mathbf{k}}(\xi) \hat{H}_{L+1, L}^{\mathbf{k}},
$$

where $\hat{G}_{L+1, L+1}^{\mathbf{k}}(\xi)$ denotes the surface element of the Green's function of the semi-infinite substrate obtained by removing the surface layers with $1 \leqslant l \leqslant L$. In Ref. [26] we derived another expression for the embedding potential,

$$
\hat{\Sigma}_{L, L}^{\mathbf{k}}(\xi)=\hat{H}_{01}^{b, \mathbf{k}} \hat{V} \hat{Q} \hat{V}^{-1},
$$

which holds true provided $\hat{H}_{l, l^{\prime}}^{\mathbf{k}}\left(l, l^{\prime}>L\right)$ and $\hat{H}_{L, L+1}^{\mathbf{k}}$, the transfer matrix at the boundary, become identical with the bulk Hamiltonian matrices, $\hat{H}_{00}^{b, \mathbf{k}}$ and $\hat{H}_{01}^{b, \mathbf{k}}$. In Eq. (13), $\hat{V}$ and $\hat{Q}$ are $N \times N$ matrices defined by $\hat{V}=\left(\mathbf{v}_{1}, \mathbf{v}_{2}, \ldots, \mathbf{v}_{N}\right)$ and $\hat{Q}=\operatorname{diag}\left(e^{i q_{1}}, e^{i q_{2}}, \ldots, e^{i q_{N}}\right)$. Here, $\mathbf{v}_{i}(i=1$ to $N)$ are the eigenvectors of the generalized Bloch states in the bulk crystal with energy $\xi$ and wave vector $\left(\mathbf{k}, q_{i}\right)$, which either propagate or decay toward the interior of the substrate.

Now, let us consider a bulk crystal with both time reversal and space inversion symmetries. Then, as discussed in Sec. II B, the evanescent waves for a real energy $\varepsilon$ in a surface-projected bulk band gap are made out of $N / 2$ doubly degenerate pairs of evanescent waves, and thus $\hat{V}$ and $\hat{Q}$ are written as

$$
\begin{aligned}
& \hat{V}=\left(\mathbf{v}_{1}, \ldots, \mathbf{v}_{N / 2}, \hat{\mathcal{T}} \hat{\mathcal{P}} \mathbf{w}_{1}, \ldots, \hat{\mathcal{T}} \hat{\mathcal{P}} \mathbf{w}_{N / 2}\right), \\
& \hat{Q}=\operatorname{diag}\left(e^{i q_{1}}, \ldots, e^{i q_{N / 2}}, e^{i q_{1}}, \ldots, e^{i q_{N / 2}}\right) .
\end{aligned}
$$

In the presence of SOI, the two evanescent waves, $\left\{\mathbf{v}_{i}, \hat{\mathcal{T}} \hat{\mathcal{P}} \mathbf{w}_{i}\right\}$ $(i=1,2, \ldots, N / 2)$, become two distinct solutions of the Schrödinger equation that do not satisfy the same boundary condition. Since the embedding potential constructed by Eq. (13) contains the full information on both eigenvectors, it can describe the contributions of the substrate layers with $l>L$ to the Rashba spin splitting of the surface bands.

\section{MODEL CALCULATION}

\section{A. Hamiltonian}

As an example to demonstrate the properties of the complex energy bands discussed in the preceding section, we consider an $s p$-band tight-binding Hamiltonian on a square lattice, where the sides of its square unit cell are chosen as the $x$ and $z$ axes, and the $y$ axis is normal to the square plane. To expand electron wave function, we use $s, p_{x}, p_{y}$, and $p_{z}$ orbitals on each atomic site. We denote the onsite energies of the four atomic orbitals by $\left\{\varepsilon_{s}^{a}, \varepsilon_{x}^{a}, \varepsilon_{y}^{a}, \varepsilon_{z}^{a}\right\}$, the nearestneighbor hopping integrals for the $\sigma$ bond by $\left\{t_{s s}^{\sigma}, t_{s p}^{\sigma}, t_{p p}^{\sigma}\right\}$, the nearest-neighbor hopping integral for the $\pi$ bond by $t_{p p}^{\pi}$, and the spin-orbit coupling constant for $p$ orbitals by $\lambda$. As mentioned in the Introduction, the SOI on each atomic site is given by $\hat{H}_{s o}=2 \lambda(\vec{l} \cdot \vec{s})$.

To expand generalized Bloch states, we employ LCAO type basis functions, $\left|\chi_{l n}^{k_{x}}\right\rangle$ 's, specified by layer (atomic chain running in the $x$ direction) index $l$, the wave number in the $x$ direction $k_{x}$, and composite index $n$ representing both orbital type and spin directions $(N=8)$. The present system possesses not only time reversal and space inversion symmetries but also mirror reflection symmetry about the $x z$ plane. By choosing the eigenstates of $\hat{s}_{y}$ (the $y$ component of the electron spin operator) as the spin part of the basis functions, the basis functions become eigenstates of the mirror reflection with eigenvalues $M= \pm i$. They are divided into two subsets having opposite mirror parities. The basis set for mirror parity $M=-i$ is given by $\left\{\left|\chi_{l, s \uparrow}^{k_{x}}\right\rangle,\left|\chi_{l, p_{z} \uparrow}^{k_{x}}\right\rangle,\left|\chi_{l, p_{x} \uparrow}^{k_{x}}\right\rangle,\left|\chi_{l, p_{y} \downarrow}^{k_{x}}\right\rangle\right\}$, whereas that for $M=+i$ is given by $\left\{\left|\chi_{l, s \downarrow}^{k_{x}}\right\rangle,\left|\chi_{l, p_{z} \downarrow}^{k_{x}}\right\rangle,\left|\chi_{l, p_{x} \downarrow}^{k_{x}}\right\rangle,\left|\chi_{l, p_{y} \uparrow}^{k_{x}}\right\rangle\right\}$. With this basis set, the original $8 \times 8$ Hamiltonian is divided into two diagonal blocks. The $4 \times 4$ complex Hamiltonian defined by Eq. (6) for each block is written as

$$
\begin{aligned}
& \hat{\mathcal{H}}\left(k_{x}, q, M=\mp i\right) \\
& \quad=\left(\begin{array}{cccc}
\varepsilon_{s}\left(k_{x}, q\right) & 2 i t_{s p}^{\sigma} \sin q & 2 i t_{s p}^{\sigma} \sin k_{x} & 0 \\
-2 i t_{s p}^{\sigma} \sin q & \varepsilon_{z}\left(k_{x}, q\right) & \mp i \lambda & \pm \lambda \\
-2 i t_{s p}^{\sigma} \sin k_{x} & \pm i \lambda & \varepsilon_{x}\left(k_{x}, q\right) & -i \lambda \\
0 & \pm \lambda & +i \lambda & \varepsilon_{y}\left(k_{x}, q\right)
\end{array}\right),
\end{aligned}
$$

with

$$
\begin{aligned}
& \varepsilon_{s}\left(k_{x}, q\right)=\varepsilon_{s}^{a}+2\left(t_{s s}^{\sigma} \cos k_{x}+t_{s s}^{\sigma} \cos q\right), \\
& \varepsilon_{z}\left(k_{x}, q\right)=\varepsilon_{z}^{a}+2\left(t_{p p}^{\pi} \cos k_{x}+t_{p p}^{\sigma} \cos q\right), \\
& \varepsilon_{x}\left(k_{x}, q\right)=\varepsilon_{x}^{a}+2\left(t_{p p}^{\sigma} \cos k_{x}+t_{p p}^{\pi} \cos q\right), \\
& \varepsilon_{y}\left(k_{x}, q\right)=\varepsilon_{y}^{a}+2\left(t_{p p}^{\pi} \cos k_{x}+t_{p p}^{\pi} \cos q\right) .
\end{aligned}
$$

From Eq. (15), it is easy to check that $\operatorname{det}\left[\hat{\mathcal{H}}\left(k_{x}, q, M\right)-\right.$ $\varepsilon \hat{I}]$ is identical for both $M$ values, so that complex energy bands determined by Eq. (7) are doubly degenerate with respect to mirror parities. Indeed, if we denote the eigenvector with complex wave vector $\left(k_{x}, q\right)$ and $M=-i$ by $\mathbf{v}=$ $\left(v_{s}, v_{z}, v_{x}, v_{y} ; 0,0,0,0\right)$ (the former and latter four elements 

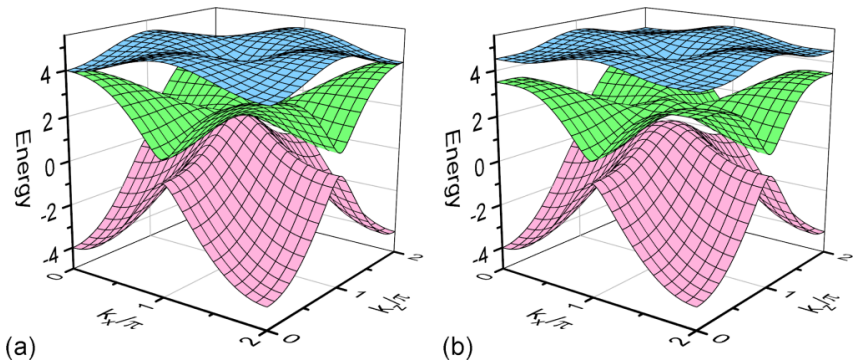

FIG. 2. Energy dispersion with $\left(k_{x}, k_{z}\right)$ of bulk Bloch states for the three-band model Hamiltonian on a square lattice. (a) $\lambda=0$ and (b) $\lambda=0.5$. The other parameters common to both panels are $\varepsilon_{s}^{a}=0$, $\varepsilon_{p}^{a}=3, t_{s s}^{\sigma}=-1.0, t_{p p}^{\sigma}=0.75, t_{p p}^{\pi}=-0.25$, and $t_{s p}^{\sigma}=1.0$.

correspond to $M=-i$ and $+i$ ) and its corresponding left eigenvector $\mathbf{w}=\left(w_{s}, w_{z}, w_{x}, w_{y} ; 0,0,0,0\right)$, it is straightforward to show that $\hat{\mathcal{T}} \hat{\mathcal{P}}_{\mathbf{w}}=\left(0,0,0,0 ; w_{s}^{*},-w_{z}^{*},-w_{x}^{*}, w_{y}^{*}\right)$ is the eigenvector with the same complex wave vector $\left(k_{x}, q\right)$ and the opposite mirror parity $M=+i$. As for evanescent states, there is no simplified operator relating these two solutions when $|\lambda|>0$.

\section{B. Shockley surface bands in the weak SOI regime}

We present a model calculation of the Rashba spin splitting by using the tight-binding Hamiltonian introduced in the preceding section. In the present section, we treat bulk crystals with weak SOIs, while we treat a topological insulator with strong SOIs in the next section. For simplicity, we discard the $p_{y}$ orbital from Eq. (15). The resulting three-band $\left(s, p_{z}, p_{x}\right)$ model possesses the $y$ component of the electron spin as a quantum number.

As an example, we set the Hamiltonian parameters to be $\varepsilon_{s}^{a}=0, \varepsilon_{x}^{a}=\varepsilon_{z}^{a}=3$ (denoted by $\varepsilon_{p}^{a}$ hereafter), $t_{s s}^{\sigma}=-1.0$, $t_{p p}^{\sigma}=0.75, t_{p p}^{\pi}=-0.25$, and $t_{s p}^{\sigma}=1.0$, while $\lambda$ is varied between 0 and 0.5. In Fig. 2 we show the energy dispersion with $\left(k_{x}, k_{z}\right)$ of the three Bloch bands in the 2D Brillouin zone for (a) $\lambda=0$ and (b) $\lambda=0.5$. Their energies are given by $\left\{\varepsilon_{s}^{a}+\right.$ $\left.4 t_{s s}^{\sigma}, \varepsilon_{p}^{a}+2\left(t_{p p}^{\sigma}+t_{p p}^{\pi}\right) \pm \lambda\right\} \quad$ at $\left(k_{x}, k_{z}\right)=(0,0), \quad\left\{\varepsilon_{s}^{a}, \varepsilon_{p}^{a} \pm\right.$ $\sqrt{\left.4\left(t_{p p}^{\sigma}-t_{p p}^{\pi}\right)^{2}+\lambda^{2}\right\}}$ at $\left(k_{x}, k_{z}\right)=(\pi, 0)$ and $(0, \pi)$, and $\left\{\varepsilon_{s}^{a}-4 t_{s s}^{\sigma}, \varepsilon_{p}^{a}-2\left(t_{p p}^{\sigma}+t_{p p}^{\pi}\right) \pm \lambda\right\}$ at $\left(k_{x}, k_{z}\right)=(\pi, \pi)$. The aforementioned parameter set is chosen such that (i) the two upper bands have the $p$-orbital character while the lowest one has the $s$-orbital character except near the zone boundary $\left(k_{x}, k_{z}\right)=(\pi, \pi)$, (ii) the band inversion occurs at $(\pi, \pi)$, i.e., the energy of the $s$ state, $\varepsilon_{s}^{a}-4 t_{s s}^{\sigma}=4$, is higher than the corresponding ones of the $p$ states, $\varepsilon_{p}^{a}-2\left(t_{p p}^{\sigma}+t_{p p}^{\pi}\right) \pm \lambda=$ $2 \pm \lambda$, and (iii) a finite band gap is opened between the highest and second highest bands for $\lambda>0$. The relatively large value of $t_{s p}^{\sigma}$ and the opposite signs of $t_{p p}^{\sigma}$ and $t_{p p}^{\pi}$ are favorable for (iii), and then, (ii) ensures that a surface band emerges within the band gap between the highest and second highest band gap as indicated by the parity analysis of the three bulk bands at the four time-reversal invariant momentum (TRIM) points [31] in the 2D Brillouin zone.

We calculate the Green's function of the semi-infinite crystal whose surface (one-dimensional edge) is parallel to the $x$ direction via the embedding method described in Sec. II C.
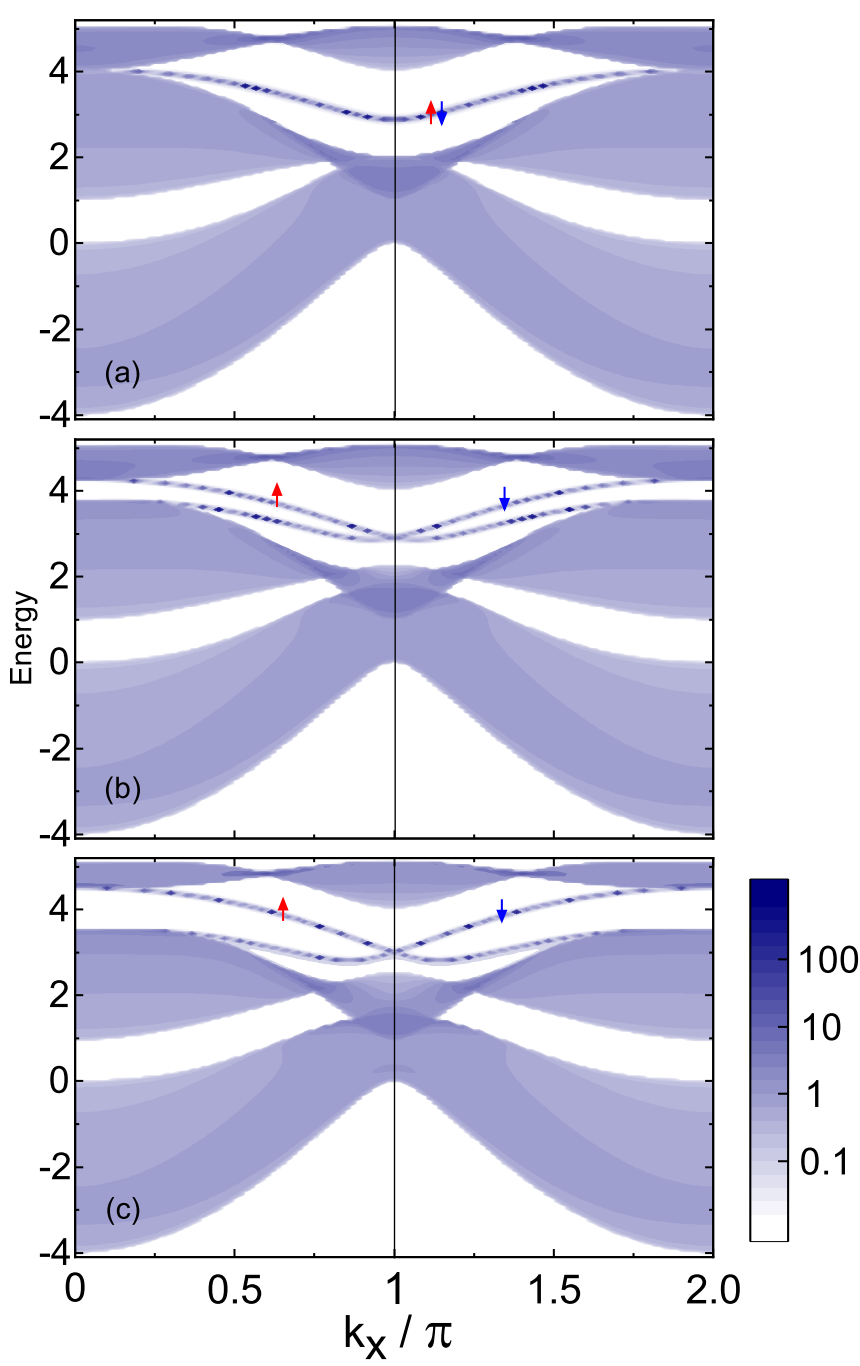

FIG. 3. Intensity plot of the $k_{x}$-resolved DOS of the outermost layer, $\rho_{1}\left(k_{x}, \varepsilon\right)$, for semi-infinite surfaces with (a) $\lambda=0$, (b) $\lambda=$ 0.25 , and (c) $\lambda=0.5$. The other parameters are the same as those in Fig. 2. See the vertical color scale for the actual DOS values. Spin-up and spin-down surface bands are indicated by red and blue arrows, respectively. Shaded regions represent surface-projected bulk bands. Imaginary energy $\gamma=5 \times 10^{-4}$.

We consider a simplified case in which the Hamiltonian matrices in the surface region are the same as those in the bulk including the outermost layer $(l=1)$. This model surface is called "bulk truncated surface" in the present work. While this model may not represent a realistic surface, it is useful in discussing the properties of the evanescent states in the bulk region, since the surface-state wave functions of this model are expressed as a superposition of the bulk evanescent waves up to the outermost layer.

We define the $k_{x}$-resolved density of states (DOS) of the $l$ th layer by

$$
\rho_{l}\left(k_{x}, \varepsilon\right)=-\frac{1}{\pi} \operatorname{Im}\left[\operatorname{Tr} \hat{G}_{l, l}^{k_{x}}(\varepsilon+i \gamma)\right],
$$

where we add a small imaginary energy $\gamma$, which enables us to treat both bulk and surface states in the same manner when plotting their DOS, since the $\delta$-function-like DOS peaks of the 
surface states are broadened. In Fig. 3 we show the intensity plot of the $k_{x}$-resolved DOS of the outermost layer, $\rho_{1}\left(k_{x}, \varepsilon\right)$, for three $\lambda$ values. When $\lambda=0$ [panel (a)], the surface band is degenerate with respect to spin, since the Hamiltonian matrices for both spin components are the same. Now, we switch on the SOI by increasing $\lambda$ [panels (b) and (c)]. Since the signs of the $(2,3)$ and $(3,2)$ elements of Eq. (15) are opposite with respect to two spin directions, $\sigma_{y}=+1(\uparrow),-1(\downarrow)$, the evanescent waves at a given $\left(k_{x}, \varepsilon\right)$ for both spin components become distinct solutions, as a result of which the degeneracy of surface bands is lifted except at the TRIM point, $k_{x}=\pi$. The splitting at small $\Delta k_{x}=k_{x}-\pi$ is proportional to $\Delta k_{x}$ with its constant of proportionality (the Rashba parameter) increasing with increasing $\lambda$.

The aforementioned degeneracy lifting arises because the linear change in surface-state wave functions upon introducing $\hat{H}_{s o}$ differs between both spin directions. Nevertheless, interestingly, the energy splitting itself can be estimated from the wave functions for $\lambda=0$ via the first-order perturbation theory with respect to $\hat{H}_{s o}$. When $\lambda=0$, the surface-state wave function at $k_{x}=\pi$ is a linear combination of only $s$ and $p_{z}$ orbitals, while they mix with $p_{x}$ when $k_{x}$ deviates from $\pi$. Hence, the three orbital components of the surfacestate wave function $\left|\phi_{\sigma_{y}}\right\rangle$ on layer $l$ can be expressed as $\left(\alpha_{s}(l), \alpha_{z}(l), \beta_{x}(l) \Delta k_{x}\right)$ when $\Delta k_{x} \ll 1$, where $\alpha_{s}(l), \alpha_{z}(l)$, and $\beta_{x}(l)$ are spin- and $k_{x}$-independent functions of $l$ that decay with increasing $l$. Then, the first-order perturbation theory suggests that the change in surface-state energies for small $\lambda$ is estimated as

$$
\begin{gathered}
\Delta \varepsilon_{s}\left(k_{x}, \sigma_{y}= \pm 1\right)=\left\langle\phi_{\sigma_{y}}\left|\hat{H}_{s o}\right| \phi_{\sigma_{y}}\right\rangle \\
= \pm 2 \lambda \Delta k_{x} \sum_{l \geqslant 1} \operatorname{Im}\left[\alpha_{z}^{*}(l) \beta_{x}(l)\right]
\end{gathered}
$$

which agrees with the behavior of the spin splitting of two surface bands around $k_{x}=\pi$ in Fig. 3. Equation (18) indicates that the spin splitting is caused by the mixing of two $p$ orbitals in surface-state wave functions. This is similar to the Rashba spin splitting of the $L$-gap surface states on noblemetal surfaces $[1,7,8]$ where the energy splitting occurs via the mixing of two $d$ orbitals in surface-state wave functions [19,32].

\section{Topological surface bands in the strong SOI regime}

When the spin-orbit coupling constant $\lambda$ is large, it is more convenient to rewrite the upper $3 \times 3$ block of the complex Hamiltonian (15) by using basis functions $| \pm 1\rangle=\left(\left|p_{z}\right\rangle \pm\right.$ $\left.i\left|p_{x}\right\rangle\right) / \sqrt{2}$, which are the eigenfunctions of the $y$ component of orbital angular momentum with eigenvalues $m= \pm 1$. We adopt the basis set $\left\{\left|\chi_{l, s, \uparrow}^{k_{x}}\right\rangle,\left|\chi_{l,-1, \uparrow}^{k_{x}}\right\rangle,\left|\chi_{l,+1, \uparrow}^{k_{x}}\right\rangle\right\}$ for $\sigma_{y}=1(\uparrow)$, and $\left\{\left|\chi_{l, s, \downarrow}^{k_{x}}\right\rangle,\left|\chi_{l,+1, \downarrow}^{k_{x}}\right\rangle,\left|\chi_{l,-1, \downarrow}^{k_{x}}\right\rangle\right\}$ for $\sigma_{y}=-1(\downarrow)$. Then, the corresponding complex Hamiltonian for both spin directions $\sigma_{y}= \pm 1$ is written as

$$
\hat{\mathcal{H}}\left(k_{x}, q, \sigma_{y}= \pm 1\right)=\left(\begin{array}{c}
\varepsilon_{s}\left(k_{x}, q\right) \\
\sqrt{2} t_{s p}^{\sigma}\left(-i \sin q \pm \sin k_{x}\right) \\
\sqrt{2} t_{s p}^{\sigma}\left(-i \sin q \mp \sin k_{x}\right)
\end{array}\right.
$$

$$
\left.\begin{array}{c}
\sqrt{2} t_{s p}^{\sigma}\left(i \sin q \mp \sin k_{x}\right) \\
\delta \varepsilon\left(k_{x}, q\right) \\
\varepsilon_{j=3 / 2}\left(k_{x}, q\right)
\end{array}\right)
$$

with

$$
\begin{gathered}
\varepsilon_{j=1 / 2}\left(k_{x}, q\right)=\frac{\varepsilon_{z}^{a}+\varepsilon_{x}^{a}}{2}+\left(t_{p p}^{\sigma}+t_{p p}^{\pi}\right)\left(\cos k_{x}+\cos q\right)-\lambda, \\
\varepsilon_{j=3 / 2}\left(k_{x}, q\right)=\frac{\varepsilon_{z}^{a}+\varepsilon_{x}^{a}}{2}+\left(t_{p p}^{\sigma}+t_{p p}^{\pi}\right)\left(\cos k_{x}+\cos q\right)+\lambda, \\
\delta \varepsilon\left(k_{x}, q\right)=\frac{\varepsilon_{z}^{a}-\varepsilon_{x}^{a}}{2}+\left(t_{p p}^{\pi}-t_{p p}^{\sigma}\right)\left(\cos k_{x}-\cos q\right),
\end{gathered}
$$

where $j$ is defined by $j=\left|m+\sigma_{y}\right|$. If the $s$ band and one of the $p$ bands with $j=\frac{1}{2}$ or $\frac{3}{2}$ are energetically close to each other while the other $p$ band is energetically far from the former two bands, one may discard the latter $p$ band from Eq. (19). The resultant two-band model coincides with the Bernevig-Hughes-Zhang model for $2 \mathrm{D}$ topological insulators $[24,33,34]$.

As an example, we calculate the electronic structure of the bulk truncated surface, which is parallel to the $x$ direction, for the parameter set, $\varepsilon_{s}^{a}=0, \varepsilon_{z}^{a}=\varepsilon_{x}^{a}=4, t_{s s}^{\sigma}=-1.0, t_{p p}^{\sigma}=$ $t_{p p}^{\pi}=0.75, t_{s p}^{\sigma}=0.75$, and $\lambda=2.0$. This parameter set is chosen such that (i) $\delta \varepsilon$ becomes identically zero, i.e., the two $p$ bands are separated energetically by a constant $2 \lambda$ over the whole Brillouin zone if $t_{s p}^{\sigma}=0$, (ii) the three bands are of the $s, j=\frac{1}{2}$, and $j=\frac{3}{2}$ character in the ascending order

except near the zone boundary, (iii) the band inversion occurs at $\left(k_{x}, k_{z}\right)=(\pi, \pi)$, where the $s$ state has the highest energy, $\varepsilon_{s}^{a}-4 t_{s s}^{\sigma}=4$, and (iv) a finite band gap is opened in the whole $2 \mathrm{D}$ Brillouin zone between two neighboring bands. The parity analysis of the three bands at the four TRIM points in the 2D Brillouin zone [31] reveals that the present system is a 2D topological insulator with regard to both the lower and higher band gaps. In Fig. 4 we show the intensity plot of the $k_{x}$-resolved DOS of the outermost layer, $\rho_{1}\left(k_{x}, \varepsilon\right)$. As is seen, a pair of topological surface bands crossing the band gap and having opposite spin directions emerge in each of the two surface-projected bulk band gaps.

The spin splitting of the two surface bands in the lower (higher) band gap occurs because the sign in front of $\sqrt{2} t_{s p}^{\sigma} \sin k_{x}$ in the $(1,2)$ and $(2,1)[(1,3)$ and $(3,1)]$ elements of the complex Hamiltonian (19) differs between the two spin components. This results in spin-dependent modifications of the evanescent-state wave functions. While the spin splitting of surface bands in Fig. 4 may look similar to those in Figs. 3(b) and 3(c), there is a crucial difference in its nature. That is, in the case of Fig. 3, the magnitude of the energy splitting and spin texture of both surface bands are determined by $\lambda$. This allowed us to treat the spin splitting of surface bands by first-order perturbation theory with respect to $\hat{H}_{s o}$. On the 


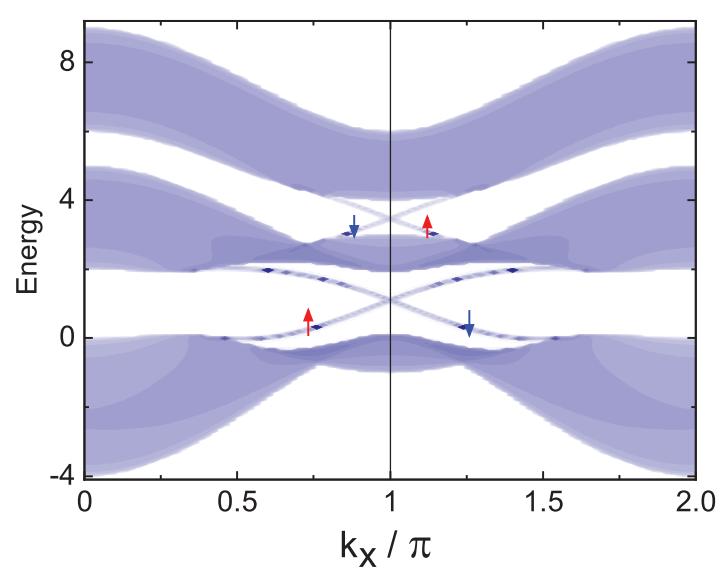

FIG. 4. Intensity plot of the $k_{x}$-resolved DOS of the outermost layer, $\rho_{1}\left(k_{x}, \varepsilon\right)$, for a semi-infinite surface with Hamiltonian parameters, $\varepsilon_{s}^{a}=0, \varepsilon_{z}^{a}=\varepsilon_{x}^{a}=4, t_{s s}^{\sigma}=-1.0, t_{p p}^{\sigma}=t_{p p}^{\pi}=0.75, t_{s p}^{\sigma}=0.75$, and $\lambda=2.0$. The color scaling of the DOS is the same as that in Fig. 3. Spin-up and spin-down surface bands are indicated by red and blue arrows, respectively. Shaded regions represent surface-projected bulk bands. Imaginary energy $\gamma=5 \times 10^{-4}$.

other hand, in the present case, it is the term $\pm \sqrt{2} t_{s p}^{\sigma} \sin k_{x}$ in the $(1,2)$ and $(2,1)$ elements of Eq. (19) (as for the surface bands in the lower band gap), or the term $\mp \sqrt{2} t_{s p}^{\sigma} \sin k_{x}$ in the $(1,3)$ and $(3,1)$ elements (as for the surface bands in the higher band gap) that governs the energy splitting of two surface bands. The sign of this term in the $(1,2)$ and $(2,1)$ elements is opposite to that in the $(1,3)$ and $(3,1)$ elements. This explains why the spin polarization of the two surface bands in the lower band gap is opposite to the corresponding one in the higher band gap.

\section{SUMMARY}

In the present paper we have shed light on the role of the bulk complex energy bands in the Rashba spin splitting of Shockley surface states. According to the embedding theory, the effects of the bulk potential region on the surface electronic structure is represented by the embedding potential generated from the wave functions of complex energy bands. Since the complex energy bands are doubly degenerate in a bulk crystal with both time reversal and space inversion symmetries, one might wonder why the bulk region can contribute to the Rashba spin splitting of Shockley surface bands. In the present paper, we have shown that while two states in a degenerate pair are related to each other by operator $\hat{\mathcal{T}} \hat{\mathcal{P}}$ with regard to propagating Bloch states, the evanescent states forming a degenerate pair become two distinct solutions of the Schrödinger equation in the presence of spin-orbit interaction, which are not related by a simplified mathematical operator and also do not satisfy the same boundary condition at the boundary surface between the surface and bulk regions. Since the tail of the surface-state wave function is expressed as a superposition of the evanescent waves, the bulk region also contributes to the Rashba spin splitting. In the embedding theory, this contribution is expressed by the embedding potential that contains the full information on the wave functions of the evanescent states. We have illustrated this effect by a DFT calculation for the $\mathrm{Au}(111)$ surface and also by using a simplified $s p$-band model Hamiltonian on a square lattice.

\section{ACKNOWLEDGMENTS}

This work is supported by the Japan Society for the Promotion of Science (JSPS) KAKENHI, Grant No. JP18H01875. The author is grateful to the Alexander von Humboldt foundation for support during his stay in Jülich.
[1] S. LaShell, B. A. McDougall, and E. Jensen, Phys. Rev. Lett. 77, 3419 (1996).

[2] Y. A. Bychkov and E. I. Rashba, JETP Lett. 39, 78 (1984).

[3] L. Petersen and P. Hedegård, Surf. Sci. 459, 49 (2000).

[4] G. Nicolay, F. Reinert, S. Hüfner, and P. Blaha, Phys. Rev. B 65, 033407 (2001).

[5] Yu. M. Koroteev, G. Bihlmayer, J. E. Gayone, E. V. Chulkov, S. Blügel, P. M. Echenique, and Ph. Hofmann, Phys. Rev. Lett. 93, 046403 (2004).

[6] K. Miyamoto, H. Miyahara, K. Kuroda, T. Maegawa, A. Kimura, and T. Okuda, Phys. Rev. B 97, 085433 (2018).

[7] A. Tamai, W. Meevasana, P. D. C. King, C. W. Nicholson, A. de la Torre, E. Rozbicki, and F. Baumberger, Phys. Rev. B 87, 075113 (2013).

[8] K. Yaji, A. Harasawa, K. Kuroda, R. Li, B. Yan, F. Komori, and S. Shin, Phys. Rev. B 98, 041404(R) (2018).

[9] C. R. Ast, J. Henk, A. Ernst, L. Moreschini, M. C. Falub, D. Pacilé, P. Bruno, K. Kern, and M. Grioni, Phys. Rev. Lett. 98, 186807 (2007).

[10] G. Bihlmayer, S. Blügel, and E. V. Chulkov, Phys. Rev. B 75, 195414 (2007).
[11] F. Meier, H. Dil, J. Lobo-Checa, L. Patthey, and J. Osterwalder, Phys. Rev. B 77, 165431 (2008).

[12] R. Noguchi, K. Kuroda, K. Yaji, K. Kobayashi, M. Sakano, A. Harasawa, T. Kondo, F. Komori, and S. Shin, Phys. Rev. B 95, 041111(R) (2017).

[13] G. Bihlmayer, Yu. M. Koroteev, P. M. Echenique, E. V. Chulkov, and S. Blügel, Surf. Sci. 600, 3888 (2006).

[14] M. Nagano, A. Kodama, T. Shishidou, and T. Oguchi, J. Phys.: Condens. Matter 21, 064239 (2009).

[15] S. R. Park, C. H. Kim, J. Yu, J. H. Han, and C. Kim, Phys. Rev. Lett. 107, 156803 (2011).

[16] B. Kim, C. H. Kim, P. Kim, W. Jung, Y. Kim, Y. Koh, M. Arita, K. Shimada, H. Namatame, M. Taniguchi, J. Yu, and C. Kim, Phys. Rev. B 85, 195402 (2012).

[17] E. E. Krasovskii, Phys. Rev. B 90, 115434 (2014).

[18] B. Yan, B. Stadtmüller, N. Haag, S. Jakobs, J. Seidel, D. Jungkenn, S. Mathias, M. Cinchetti, M. Aeschlimann, and C. Felser, Nat. Commun. 6, 10167 (2015).

[19] H. Ishida, Phys. Rev. B 90, 235422 (2014).

[20] D. J. Singh and L. Nordstrom, Planewaves, Pseudopotentials, and the LAPW Method, 2nd ed. (Springer, New York, 2006). 
[21] J. E. Inglesfield, J. Phys. C 14, 3795 (1981).

[22] J. E. Inglesfield, The Embedding Method for Electronic Structure (IOP publishing, Bristol, 2015).

[23] D. Wortmann, H. Ishida, and S. Blügel, Phys. Rev. B 65, 165103 (2002).

[24] X. Dang, J. D. Burton, A. Kalitsov, J. P. Velev, and E. Y. Tsymbal, Phys. Rev. B 90, 155307 (2014).

[25] J. Betancourt, S. Li, X. Dang, J. D. Burton, E. Y. Tsymbal, and J. P. Velev, J. Phys.: Condens. Matter 28, 395501 (2016).

[26] H. Ishida, A. Liebsch, and D. Wortmann, Phys. Rev. B 96, 125413 (2017).

[27] I. Rungger and S. Sanvito, Phys. Rev. B 78, 035407 (2008).
[28] J. C. Y. Teo, L. Fu, and C. L. Kane, Phys. Rev. B 78, 045426 (2008).

[29] M. Brandbyge, J. L. Mozos, P. Ordejón, J. Taylor, and K. Stokbro, Phys. Rev. B 65, 165401 (2002).

[30] Y. Xue and M. A. Ratner, Phys. Rev. B 68, 115406 (2003).

[31] L. Fu and C. L. Kane, Phys. Rev. B 76, 045302 (2007).

[32] H. Lee and H. J. Choi, Phys. Rev. B 86, 045437 (2012).

[33] B. A. Bernevig, T. L. Hughes, and S.-C. Zhang, Science, 314, 1757 (2006).

[34] B. A. Bernevig and T. L. Hughes, Topological Insulators and Topological Superconductors (Princeton University Press, Princeton, 2013). 